\title{
Metastasis tumour suppressor-1 and the aggressiveness of prostate cancer cells
}

\author{
NOHA MUSTAFA, TRACEY A. MARTIN and WEN G. JIANG \\ Metastasis and Angiogenesis Research Group, Cardiff University School of Medicine, Cardiff CF14 4XN, UK
}

Received November 5, 2010; Accepted November 29, 2010

DOI: $10.3892 / \mathrm{etm} .2010 .184$

\begin{abstract}
Previous studies have suggested that metastasis tumour suppressor-1 (MTSS1) plays a key role in cancer metastasis. Firstly, in this study we assessed MTSS1 expression levels in prostate cancer cell lines to reveal any changes in cell properties. Secondly, we aimed to clarify the cellular function of MTSS1 in prostate cancer cells. MTSS1 expression levels were assessed in different types of cancer cell lines through the RT-PCR analysis technique. The influence of MTSS1 was further examined via biological overexpression and knockdown in the prostate cancer cell lines. Two prostate cell lines were chosen for either knockdown or overexpression of the MTSS1 gene. The overexpression of MTSS1 in PC-3 human prostate cancer cells significantly suppressed the migratory, growth and adherence properties of the cells $(\mathrm{p}<0.01)$. By contrast, the knockdown of MTSS1 in DU-145 human prostate cancer cells dramatically enhanced these properties $(\mathrm{p}<0.001)$. We concluded that MTSS1 demonstrates the ability to play a role in controlling the metastatic nature of prostate cancer cells.
\end{abstract}

\section{Introduction}

Prostate cancer is the second leading cause of cancer-related mortality in males in the UK, with 9,900 deaths each year, and it accounts for $13 \%$ of cancer-related deaths in males. Approximately $85 \%$ of these cases involve men over 70 years of age. The mortality rate for prostate cancer peaked in the early 1990s and has now fallen to approximately 25 per 100,000 individuals at risk. In the UK, the survival rates have been improving, and the 5-year relative survival rate was $60 \%$ in the period 1993 to 1995 (1).

Metastasis is a complex multi-step process by which primary tumour cells invade adjacent tissue, enter the systemic

Correspondence to: Dr Wen G. Jiang, Metastasis and Angiogenesis Research Group, Cardiff University School of Medicine, Heath Park, Cardiff CF14 4XN, UK

E-mail: jiangw@cf.ac.uk

Key words: metastasis tumour suppressor-1, ribozyme transgenes, cell growth, cell migration, prostate cancer circulation (intravasate), translocate through the vasculature, arrest in distant capillaries, extravasate into the surrounding tissue parenchyma and finally proliferate from microscopic growths (micrometastases) into macroscopic secondary tumours. Metastases can be located in various organs and in different regions of the same organ. The organ microenvironment modifies the response of metastatic tumour cells to therapy and alters the effectiveness of anticancer agents in destroying the tumour cells without producing undesirable toxic effects. The major obstacle to treating metastasis is the biological heterogeneity of primary neoplasms and metastases. By the time of diagnosis, cancers contain multiple genetically unstable cell populations with diverse karyotypes, growth rates, cell-surface properties, antigenicities, immunogenicities, marker enzymes, sensitivity to various cytotoxic drugs and abilities to invade and produce metastasis (2).

Angiogenesis plays a key role in the pathogenesis of a variety of disorders, including cancer, proliferative retinopathies and rheumatoid arthritis. Accumulating evidence indicates that, for most tumours, the switch to an angiogenic phenotype depends upon the outcome of a balance between angiogenic stimuli and angiogenic inhibitors, both of which may be produced by tumour cells and perhaps by certain host cells (3). Growth and motility factors play an essential role in migration processes at various levels of the metastatic cascade. These factors include metastasis activators and suppressors, which act in autocrine or paracrine manners through special receptors that mediate different signals through tyrosine phosphorylation (4). However, metastasis suppressors may inhibit metastasis at any step of the metastatic cascade without blocking tumourigenicity (5).

Metastasis tumour suppressor-1 (MTSS1), also known as Missing in Metastasis (MIM), was originally identified as a tumour suppressor since it is expressed in non-metastatic, but absent from metastatic, bladder cancer cells $(6,7)$. It is expressed during development in muscles, kidneys and the liver $(8,9)$. The MTSS1 gene encodes a 5.3-kb mRNA, and it is a polypeptide of 356 amino acids with homology to the Wiscott-Aldrich Syndrome protein family (6). The MIM protein and MIM-B, a much longer 759-amino acid protein whose $\mathrm{C}$-terminus is identical to the 356 amino acids encoded by the MIM gene (10), are cytoplasmic in location and have multidomain and scaffolding function (9).

MIM-B induces actin-rich protrusions resembling microspikes and lamellipodia at the plasma membrane and promotes 
disassembly of actin stress fibres. The actin cytoskeleton plays a key role in regulating essential cellular processes, such as endocytosis, cell migration, cytokinesis and various morphogenetic processes. In addition, MTSS1 enhances Arp2/3-mediated actin polymerization through interactions with cortactin (11). It is thus involved in cell motility and morphogenesis, and studies suggest that further analysis of MTSS1 expression or inactivation in tissue samples may define a new candidate for use as a marker for primary tumours or metastasis (12). MTSS1 is also a member of the sonic hedgehog signalling pathway, which interacts and modulates Gli responses during cell growth and carcinogenesis (8).

This study aimed to assess MTSS1 expression levels in prostate cancer cell lines in order to reveal any changes in cell properties and to clarify the cellular function of MTSS1 in these cancer cells. In the present study, we analysed MTSS1 through a series of expression and inactivation studies to clarify the function of MTSS1 in prostate cancer cells.

\section{Materials and methods}

Cell lines and culture. The cells were routinely cultured with Dulbecco's modified Eagle's medium (DMEM) (PAA Laboratories Ltd., Somerset, UK) supplemented with $10 \%$ fetal calf serum (PAA Laboratories Ltd.), penicillin and streptomycin. This study used human prostate cancer (DU-145, PC-3 and CA-HPV10) and human breast cancer (ZR-751 and MDA-MB-231) cell lines. The cell lines were purchased from the American Type Culture Collection (ATCC, Rockville, $\mathrm{MD}$, USA) and were stored at $37^{\circ} \mathrm{C}$ in $5 \% \mathrm{CO}_{2}$ and under $95 \%$ humidity.

RNA preparation and reverse transcription-polymerase chain reaction. Total cellular RNA was isolated from the homogenized human cell lines using Total RNA Isolation reagent (Advanced Biotechnologies Ltd., Epsom, Surrey, UK). RNA concentration and quality were determined through spectrophotometric measurement (WPA UV 1101; Biotech Photometer, Cambridge, UK). cDNA was generated using $200 \mathrm{ng}$ of each RNA sample and a transcription kit (Sigma, Poole, Dorset, UK) to reverse transcribe the RNA samples into cDNA. DNA quality was verified using GAPDH. MTSS1 mRNA levels were assessed using MTSS1 primers (sense, TCAAGAACAGATGGAAGAATGG; antisense, TGCGGTAGCGGTAATGTG). PCR was carried out using a T-Cy Thermocycler (Creacon Technologies Ltd., The Netherlands) and REDTaq ${ }^{\circledR}$ ReadyMix $^{\mathrm{TM}}$ PCR reaction mix (Sigma). The PCR conditions consisted of a 40-sec denaturation step $\left(95^{\circ} \mathrm{C}\right)$, a 2 -min annealing step $\left(58^{\circ} \mathrm{C}\right)$ and a 3 -min elongation step $\left(72^{\circ} \mathrm{C}\right)$; elongation took place over 36 cycles. The PCR products were next loaded onto a $0.8 \%$ agarose gel and electrophoretically separated prior to being stained with ethidium bromide and visualised under UV light.

Quantitativereal-time polymerase chainreaction.Quantitative real-time polymerase chain reaction (QPCR) is a technology that provides a broad dynamic range for detecting specific gene sequences with high sensitivity. To quantify the level of MTSS1 transcripts in the prostate cancer cell lines, the iCycler IQ system (BioRad, Camberley, UK) was used. cDNA samples were examined for MTSS1 expression using the MTSS1 QPCR primers (sense, ATATCCCAGGATGCCTTC; antisense, ACTGAACCTGACCGTACACGGTTCTCGCTTCTCTTT). The QPCR technique utilized the Amplifluor System ${ }^{\mathrm{TM}}$ (Intergen Inc., UK) and Q-PCR master mix (ABgene, Surrey, $\mathrm{UK})$, in conjunction with a universal probe (UniPrimer ${ }^{\mathrm{TM}}$ ). The real-time QPCR conditions were: $95^{\circ} \mathrm{C}$ for $15 \mathrm{~min}$, followed by 60 cycles at $95^{\circ} \mathrm{C}$ for $20 \mathrm{sec}, 55^{\circ} \mathrm{C}$ for $30 \mathrm{sec}$ and $72^{\circ} \mathrm{C}$ for $20 \mathrm{sec}$.

Generation of ribozyme transgenes and MTSS1 knockdown cells. MTSS1 expression levels were reduced in the DU-145 prostate cancer cell line using a ribozyme system. Briefly, ribozyme transgenes that specifically cleave MTSS1 mRNA were designed based on the predicted secondary structure of MTSS1. These ribozymes were then generated using touchdown PCR and subsequently cloned into the pEF6/V5-His-TOPO vector and amplified in Escherichia coli. Plasmids were purified and verified for correct size and orientation of the ribozymes, and electroporated into the DU-145 prostate cancer cell line. A closed pEF6/V5/His-TOPO plasmid (containing no ribozyme sequence) was also electroporated into the same cell line to create a control group. After selection using blasticidin, the unaltered wild-type cells were termed DU-145 WT, the wild-type cells containing closed plasmid only were termed DU-145 PEF and the wild-type cells containing plasmid with a ribozyme sequence were termed DU-145 MTSSIKD.

Generation of MTSS1-overexpressing cell lines. The full sequence of MTSS1 was amplified from cDNA using the standard PCR procedure and a master mix with a proofreading enzyme (sense primer, ATGGAGGCTGTGATTGAG; antisense, CTAAGAAAAGCGAGGGG). This MTSS1 sequence was then T-A cloned into the $\mathrm{pEF} / \mathrm{V} 5 / \mathrm{His}-\mathrm{TOPO}$ vector (Invitrogen, Paisley, UK) and then electroporated into the PC-3 prostate cancer cell line with the aim of enhancing MTSS1 expression in a cell line that does not normally express it. Multiple clones were used, assessed and sequenced. The PC-3 cells thus prepared and expressing MTSS1 were referred to in the study as PC-3 MTSS1Exp. The control group of cells contained the same plasmid vector (minus the MTSS1 sequence) and was termed PC-3 PEF.

Confirmation of MTSS1 overexpression and knockdown by Western blotting. MTSS1 protein expression was assessed in the human prostate cancer cell line lysates through standard SDS-PAGE and Western blot analysis. The cells were grown to confluence in a $75-\mathrm{cm}^{2}$ tissue culture flask before being detached using a cell scraper and pelleted. The cell pellet was then lysed in HCMF buffer with $0.5 \%$ SDS, $1 \%$ Triton X-100, $2 \mathrm{mM} \mathrm{CaCl}, 100 \mu \mathrm{g} / \mathrm{ml}$ phenylmethylsulfonyl fluoride, $1 \mathrm{mg} /$ $\mathrm{ml}$ leupeptin, $1 \mathrm{mg} / \mathrm{ml}$ aprotinin and $10 \mathrm{mM}$ sodium orthovanadate on a rotor wheel for $1 \mathrm{~h}$ and spun at 13,000 $\mathrm{x}$ g for $15 \mathrm{~min}$ to remove insolubles. The lysed protein was then quantified using the Bio-Rad DC Protein Assay kit (Bio-Rad Laboratories, CA, USA), and the samples were normalized to a standard final concentration of $1.5 \mathrm{mg} / \mathrm{ml}$ following addition of Sample Buffer, Laemmli $2 X$ concentrate (Sigma) in a 1:1 ratio. The samples were then boiled for $5 \mathrm{~min}$ before being loaded into a $10 \%$ polyacrylamide gel. Following electrophoresis, proteins were blotted 
onto a Hybond-C Extra nitrocellulose membrane (Amersham Biosciences UK Ltd., Bucks, UK), blocked in 10\% milk and subjected to specific antibody probing. The anti-MTSS1 antibody (SC-98376, Santa Cruz Biotechnologies Inc., Santa Cruz, CA, USA) was used to probe for MTSS1 at a concentration of 1:500 and anti-GAPDH (Santa Cruz Biotechnologies Inc.) at a concentration of 1:500 for GAPDH. Probing with the primary antibody was followed by probing with peroxidase-conjugated anti-rabbit (MTSS1) or anti-rabbit (GAPDH) antibody (Sigma) at a 1:1,000 concentration. The Supersignal West Dura Extended Duration substrate chemiluminescent system (Perbio Science UK Ltd., Cramlington, UK) was then used to visualize the protein bands. GAPDH expression was used as an internal control (Santa Cruz Biotechnologies, Santa Cruz, CA, USA). Protein expression was assessed and quantified using Uvitech analysis software (Uvitech, Cambridge, UK).

Tumour cell growth assay. The effects of the modification of MTSS1 expression on prostate cancer cell growth rates were assessed using an in vitro growth assay. The cells were seeded in triplicate into 96-well plates at a density of 3,000 cells/well. Plates were then incubated for 1, 3 and 5 days. Cell density was recorded after 1, 3 and 5 days by fixing cells in $4 \%$ formaldehyde, washing and staining with $0.5 \%$ (w/v) crystal violet. Following this, the crystal violet stain was extracted with $10 \%$ (v/v) acetic acid before reading the absorbance on a Bio-Tek ELx800 multiplate reader (Bio-Tek Instruments Inc., VT, USA).

Cell adhesion assay. The adhesive properties of the MTSS1-modified cells to an artificial basement membrane were quantified using the in vitro Matrigel adhesion assay. DU-145 WT, DU-145 PEF control and DU-145 MTSS1KD cells and PC-3 WT, PC-3 PEF control and PC-3 MTSS1Exp prostate cancer cells were seeded at a density of 50,000/well (in triplicate) into a 96-well plate that had been previously coated with $5 \mu \mathrm{g}$ of Matrigel artificial basement membrane. The cells were then incubated for $45 \mathrm{~min}$ to allow them to adhere before being subjected to vigorous washing $(x 4)$ in BSS to remove the non-adherent cells. The adherent cells were then fixed in $4 \%$ formaldehyde (v/v) and stained with $0.5 \%(\mathrm{w} / \mathrm{v})$ crystal violet. The number of stained adherent cells was counted in several random fields ( $\leq 40$ objective magnifications).

Wounding assay. The migratory properties of PC-3 and DU-145 cells were assessed to determine the impact of the forced expression or knockout of MTSS1 on the invasive nature of these prostate cancer cells. Cells at a density of $10^{6}$ were incubated in $10 \mathrm{ml}$ of growth medium containing $100 \mu \mathrm{l}$ of Cytodex-2 beads (GE Healthcare, Cardiff, UK) for $3 \mathrm{~h}$ to allow the cells to adhere to the beads. The beads were then washed to remove non-adherent or dead cells and resuspended in $5 \mathrm{ml}$ of medium. The cell/bead complex was then incubated in a 96-well plate overnight. Following incubation, the cells were washed with BSS, and cells that had migrated from the Cytodex- 2 beads and adhered to the base of the well were fixed in $4 \%$ formaldehyde (v/v), stained with $0.5 \%$ crystal violet (w/v) and counted under x40 objective magnification. At least five random fields were counted per well, and five duplicate wells were set up per sample. The entire experimental procedure was repeated three independent times.
Statistical analysis. All in vitro experimentation was repeated at least three independent times. The results were assessed using a two-sample, two-tailed t-test and the Minitab 14 statistical package. The values presented represent the mean \pm SEM, and $\mathrm{p} \leq 0.05$ was considered statistically significant. In vivo data were analysed using a non-parametric Mann-Whitney test, as the data did not follow a normal distribution.

\section{Results}

MTSS1 expression in human cancer cell lines and creation of prostate cancer cell sublines with differential patterns of MTSS1 expression. MTSS1 was found to be highly expressed in the DU-145 and 3T3 prostate cancer cells and at moderate levels in the CA-HPV-10 prostate and ZR 75-1 breast cancer cells, while the highly invasive prostate cancer cell line PC-3 showed little expression (Fig. 1A). The highly invasive PC-3 prostate cancer cell line was transfected with the MTSS1 expression vector. Following selection, an MTSS1-overexpressing subline was established (Fig. 1B-D). The DU-145 prostate cancer cell line was also an appropriate candidate for knockdown as the low-invasive cell line expressed levels of MTSS1. Anti-MTSS1 trangenes were used to knockdown MTSS1 expression in the DU-145 cells, followed by the establishment of a new subline which expressed a low level of MTSS1 (Fig. 1B and C).

Regulation of MTSS1 expression affects the rate of cell growth of prostate cancer cells

Knockdown of MTSS1 expression increases prostate cancer cell growth. This study found that the levels of MTSS1 protein had an effect on the growth rate of cells. The effects of the suppression of MTSS1 expression on the growth of DU-145 cells was examined following a 5-day incubation period using an in vitro cell growth assay. There was a significant difference in the cell growth rate between the wild-type (DU-145 WT) and MTSS1-suppressed (DU-145 MTSSIKD) cells. Suppression of MTSS1 expression was found to increase the cell growth rate (DU-145 MTSSIKD compared to DU-145 WT cells; p=0.001) (Fig. 2A).

Enhanced MTSS1 expression suppresses prostate cancer cell growth. The growth capacity of the prostate cancer cells following MTSS1 overexpression was examined and compared to the wild-type cells using an in vitro cell growth assay for PC-3 cell lines. A significant reduction in cell growth after 5 days following enhancement of MTSS1 expression was noted (PC-3 MTSS1Exp when compared to PC-3 WT cells; $\mathrm{p}=0.002)$ (Fig. 2B).

MTSS1 influences the adhesive ability of prostate cancer cell lines. To identify and examine the adhesive nature of the prostate cancer cells to attach to a basement membrane, an adhesion assay was carried out (Fig. 3). The results obtained show a significant increase (DU-145 MTSSIKD compared to DU-145 WT cells; $\mathrm{p}=0.001$ ) in the ability to adhere to a basement membrane (Fig. 3A, top panel), indicating that the knockdown of MTSS1 expression in this cell line resulted in a dramatic increase in the degree of adhesion. However, a significant reduction $(\mathrm{p}=0.0001)$ in adhesive ability was noted 
A

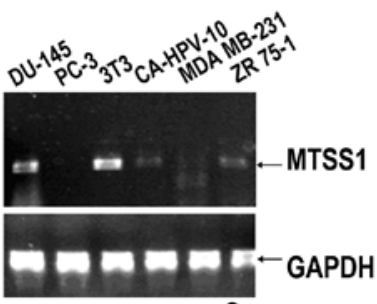

B

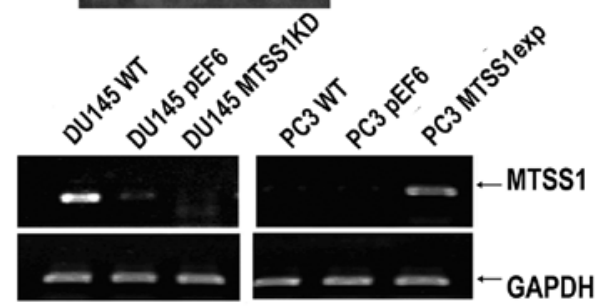

C

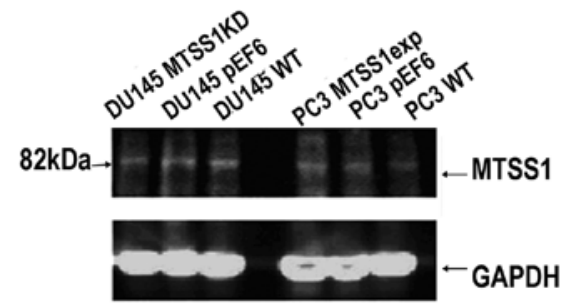

D

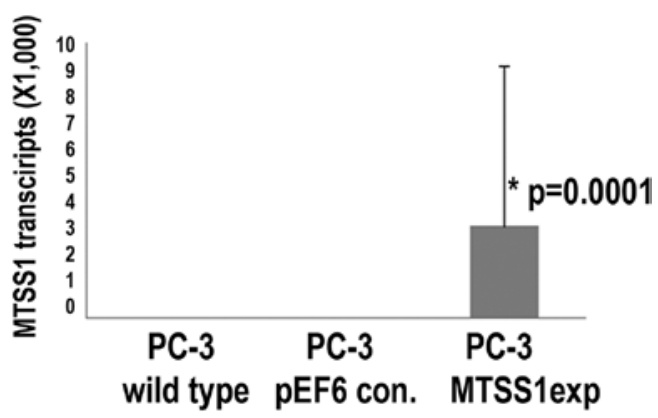

Figure 1. Expression of MTSS1 in cancer cells and creation of sublines of prostate cancer cells with differential expression of MTSS1. (A) RT-PCR analysis of MTSS1 mRNA expression in different human cancer cell lines MTSS1 was found to be highly expressed in DU-145 and 3T3 prostate cancer cells and at moderate levels in the CA-HPV-10 prostate and ZR 75-1 breast cancer cell lines, while the highly invasive prostate cancer cell line PC-3 exhibited no expression. (B) RT-PCR assessment of MTSS1 overexpression/ knockdown in the prostate cancer cell lines. MTSS1 was overexpressed in the PC-3 cell line, whereas the MTSS1 expression level was suppressed in DU-145 MTSSIKD cells. (C) Confirmation of MTSS1 protein levels by Western blotting. Results obtained at the mRNA level were confirmed at the protein level. (D) Quantitative-PCR assessment of MTSS1 overexpression. Quantification of MTSS1 transcripts in the PC-3 cell line verified the observations from the initial PCR data.

when PC-3 MTSS1Exp cells were compared to PC-3 WT cells (Fig. 3A, bottom panel).

MTSS1 has differential effects on the motile properties of the prostate cancer cell lines. The results revealed that the presence of MTSS1 had a significant impact on the motile nature of the prostate cancer cells (Fig. 3B). A significant increase $(p=0.0001)$ was observed in motility between the DU-145 MTSSIKD and DU-145 WT cells (Fig. 3B, top panel). However, forced expression of MTSS1 in the PC-3 cell line resulted in a dramatic reduction in the degree of motility (PC-3 MTSS1Exp compared to PC-3 WT cells; $\mathrm{p}=0.002$ ) (Fig. 3B, bottom).
A

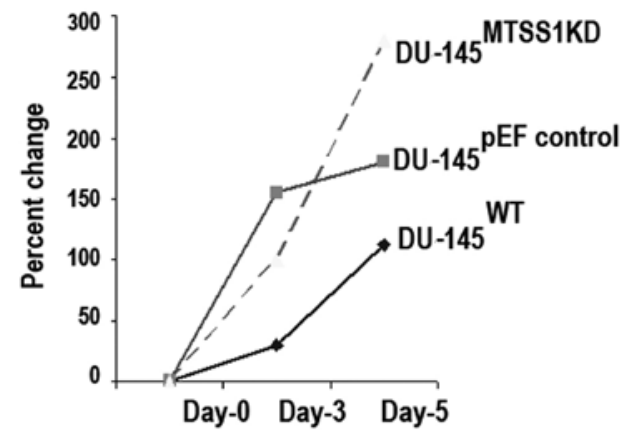

$\mathbf{B}$

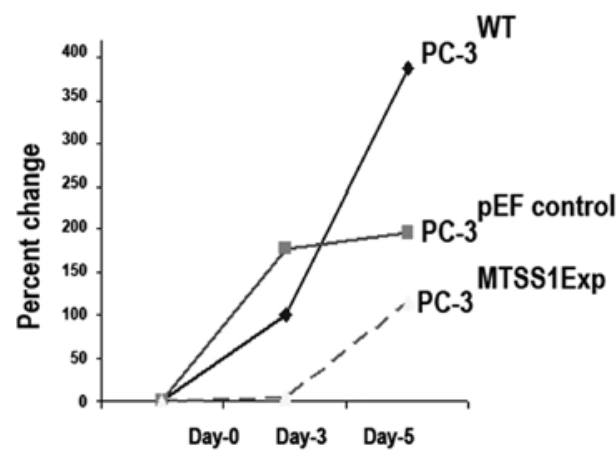

Figure 2. Growth assay demonstrating the effect of MTSS1 expression levels on prostate cancer cell growth after 5 days of incubation. (A) An increase in the DU-145 MTSS1KD cell growth rate was observed following the knockdown of MTSS1 expression ( $\mathrm{p}=0.001)$. (B) Overexpression of MTSS1 in the PC-3 cells (PC-3 MTSS1Exp) resulted in a significant decrease in the cell growth rate $(\mathrm{p}=0.002)$.

The DU-145 prostate cancer cells demonstrated motile properties in the wound closure motility assay; however, the absence of MTSS1 expression in these cells significantly increased cell migration to close the wound as compared to the DU-145 WT cells $(\mathrm{p}=0.001)$ (Fig. 4A). As shown in Fig. 4B, the presence of MTSS1 expression in the PC-3 MTSS1Exp cells significantly suppressed cell migration when compared to the extent of cell migration in the PC-3 WT cells $(\mathrm{p}=0.01)$.

\section{Discussion}

In the present study, we examined a possible association between the expression of the metastasis suppressor gene (MTSS1) and prostate tumour cell invasive behaviour. Supported by a series of cellular function tests, the present study indicates that MTSS1 acts as a powerful inhibitor to the aggressiveness of prostate cancer cells. Our initial studies examined MTSS1 expression in a variety of human normal and cancer cell lines. We reported that cancer cell lines, DU-145 and 3T3, expressed moderate levels of MTTS1.

These cell lines are considered to be of a low/non-invasive nature. The aggressive cell lines, MDA-MB-231 and PC-3, are negative for MTSS1 expression. This is of note since it may indicate that levels of MTSS1 expression are inversely correlated with aggressiveness. This is reflected in our subsequent expression modification studies, in which we created two cell models, DU-145 MTSSIKD and PC-3 MTSS1Exp, with a differential pattern of MTSS1 expression. 
A
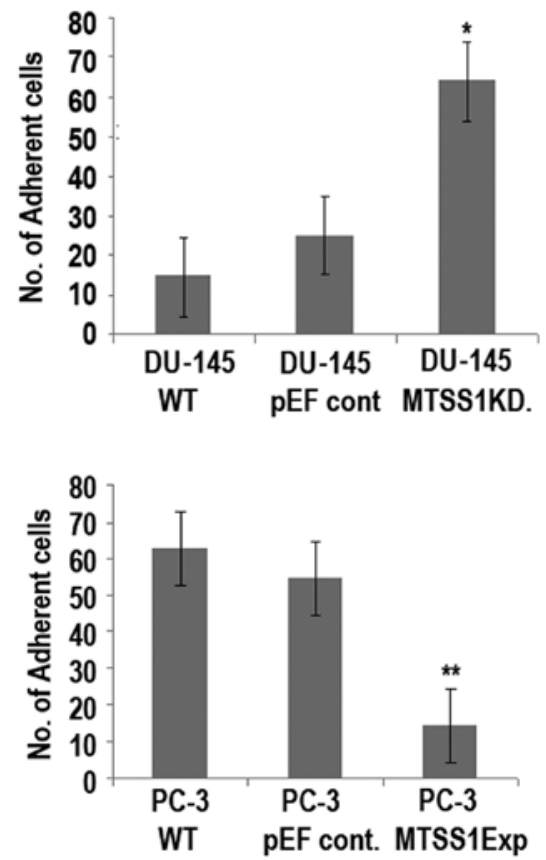
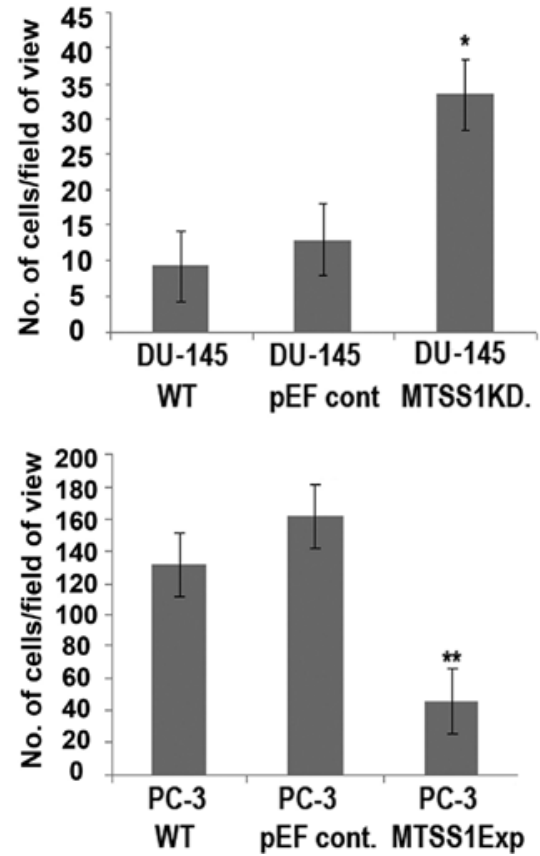

Figure 3. Adhesion and cell motility assay demonstrating the effect of MTSS1 expression levels on prostate cancer cells. (A) Suppression of MTSS1 expression levels (DU-145 MTSS1 KD) enhanced the adhesive properties of the DU-145 prostate cancer cell line ( $\mathrm{p}=0.001$ vs. control). A reduction in the adhesive nature of the PC-3 cells was observed by the forced expression of MTSS1 ( $* \mathrm{p}=0.0001 \mathrm{vs.} \mathrm{control).} \mathrm{(B)} \mathrm{Suppression} \mathrm{of} \mathrm{MTSS1} \mathrm{in} \mathrm{DU-145} \mathrm{cells} \mathrm{resulted} \mathrm{in}$ reduced motility ( $\mathrm{p}=0.0001)$. Overexpression of MTSS1 in PC-3 cells led to decreased motility $(\mathrm{p}=0.002)$.
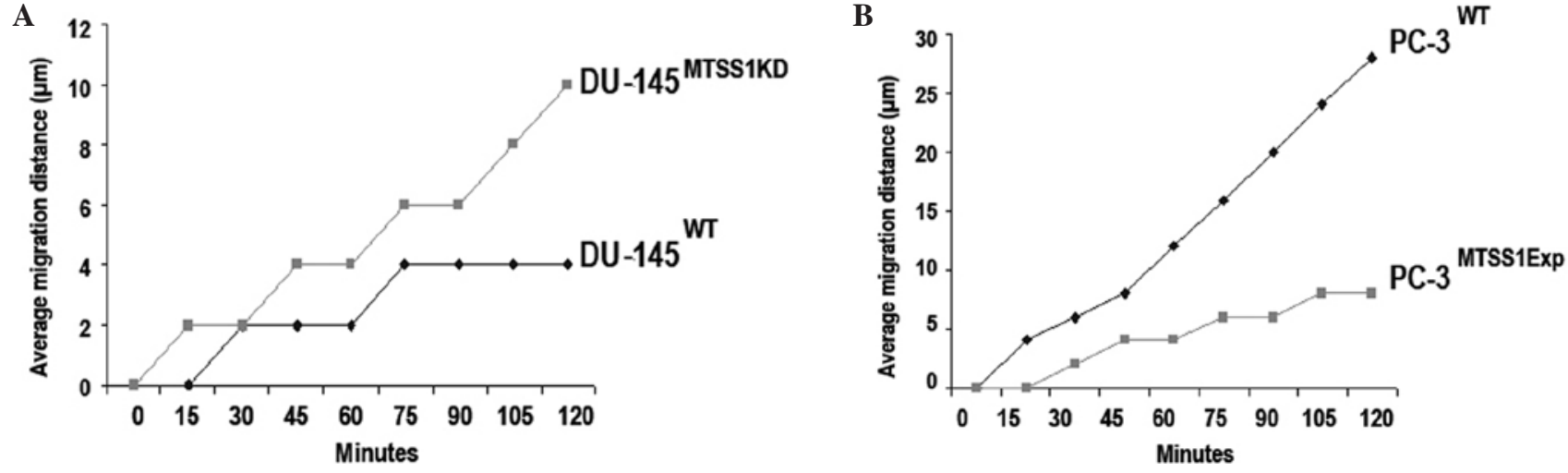

Figure 4. Migration assay demonstrating the effect of MTSS1 expression levels on prostate cancer cells. (A) An increase in the cell motility rate of DU-145 MTSSIKD cells by the knockdown of MTSS1 expression. (B) Overexpression of MTSS1 in PC-3 cells significantly reduced the migration rate.

The invasive MTSS1-negative PC-3 cell line was 'forced' to overexpress MTSS1, while the non-invasive MTSS-positive DU-145 cell line had its MTSS1 expression levels 'knocked down'. We used a range of biological function assays in vitro to assess the affect of the modification of MTSS1 expression on the metastatic nature of these prostate cancer cell lines. Our study provides evidence that the forced expression of MTSS1 in the PC-3 prostate cancer cells greatly reduced the aggressive nature of these cells by reducing their adhesive, growth and motile properties.

In contrast, the elimination of MTSS1 expression in the DU-145 cells exhibited an inverse effect. This low-invasive cell line displayed significant increases in tumour cell migration, growth and adhesion. Therefore, our findings suggest that MTSS1 plays a key role in determining the metastatic nature of prostate cancer cells.
Notably, Parr et al (13) revealed that MTSS1 acts as an indicator for survival for breast cancer patients. They demonstrated that patients expressing high levels of MTSS1 had a favourable prognosis in contrast to patients with low levels of MTSS1 expression who were associated with a poor prognosis. Utikal et al (12) suggested that the mechanism for the down-regulation of MTSS1 may involve DNA methylation. Overexpression of MTSS1 was found to enhance changes in cell shape, such as an increase in the formation of membrane ruffles and filopodia-like structures (7,10,14-16).

MTSS1 has been shown to be a metastatic suppressor in bladder cancer (6). In contrast, MTSS1 expression was found to be similar in metastatic cell lines (14) and basal cell carcinomas (9). This regulation suggests that MTSS1 levels are likely to be controlled during cell growth and development, and that MTSS1 expression is altered in cancer cells, 
leading to changes in the signalling and architecture of the cytoskeleton.

One study reported that the down-regulation of MTSS1 expression in bladder cancer may correlate with the transition of tumour cells from a distinct epithelium-like morphology to less differentiated carcinomas $(17,18)$. Another study found a dramatic increase in MTSS1 expression in normal liver specimens compared to matched hepatocellular carcinoma tumour tissue specimens (18).

Elevated MTSS1 expression was observed in early stage disease, suggesting that MTSS1 plays a key role in promoting the early development of hepatocellular carcinoma and may therefore serve as a biomarker for the prediction of early tumour development of hepatocellular carcinoma. Thus, it is likely that a metastasis suppressor for MTSS1 will be highly dependent upon tumour type (13).

In conclusion, our study indicates that MTSS1 is capable of modulating the metastatic ability in prostate cancer cells.

\section{Acknowledgements}

The authors wish to thank Cancer Research Wales for supporting their work.

\section{References}

1. Ferlay J, Parkin D and Pisani P: Estimates of the worldwide incidence of 25 major cancers in 1990. Int J Cancer 80: 158-162, 1999.

2. Fidler IJ: The pathogenesis of cancer metastasis: the 'seed and soil' hypothesis revisited. Nat Rev Cancer 3: 453-458, 2003.

3. Folkman J: Toward an understanding of angiogenesis: search and discovery. Perspect Biol Med 29: 10-36, 1985.

4. Boyer B, Valles AM and Thiery JP: Model systems of carcinoma cell dispersion. Curr Top Microbiol Immunol 213: 179-194, 1996.
5. Stafford LJ, Vaidya KS and Welch DR: Metastasis suppressor genes in cancer. Int J Biochem Cell Biol 40: 874-891, 2008.

6. Lee YG, Macoska JA, Korenchuk S and Pienta KJ: MIM, a potential metastasis suppressor gene in bladder cancer. Neoplasia 4: 291-294, 2002.

7. Loberg RD, Neeley CK and Adam-Day LL: Differential expression analysis of MIM (MTSS1) splice variants and a functional role of MIM in prostate cancer cell biology. Int J Oncol 26: $1699-1705,2005$

8. Callahan CA, Ofstad T, Horng T, Wang JK, Zhen $\mathrm{HH}$, Coulombe PA and Oro AE: MIM/BEG4, a Sonic hedgehog responsive gene that potentiates Gli-dependent transcription. Genes Dev 18: 2724-2729, 2004.

9. Mattila PK, Salminen M, Yamashiro $T$ and Lappalainen P: Mouse MIM, a tissue specific regulator of cytoskeletal dynamics, interacts with ATP-actin monomers through its C-terminal WH2 domain. J Biol Chem 278: 8452-8459, 2003.

11. Lin J, Liu J, Wang Y, Zhu J, Zhou K, Smith N and Zhan X: Differential regulation of cortactin and N-WASP-mediated actin polymerization by missing in metastasis (MIM) protein. Oncogene 24: 2059-2066, 2005.

12. Utikal J, Gratchev A and Muller-Molinet I: The expression of metastasis suppressor MIM/MTSS1 is regulated by DNA methylation. Int J Cancer 119: 2287-2293, 2006.

13. Parr C and Jiang WG: Metastasis suppressor 1 (MTSS1) demonstrates prognostic value and anti-metastatic properties in breast cancer. Int J Cancer 45: 1673-1683, 2008.

14. Liu K, Wang G, Ding $\mathrm{H}$, Chen $\mathrm{Y}$, Yu $\mathrm{G}$ and Wang J: Downregulation of metastasis suppressor 1 (MTSS1) is associated with nodal metastasis and poor outcome in Chinese patients with gastric cancer. BMC Cancer 10: 428, 2010

15. Bompard G, Sharp SJ, Freiss G and Machesky LM: Involvement of Rac in actin cytoskeleton rearrangements induced by MIM-B. J Cell Sci 118: 5393-5403, 2005.

16. Gonzalez-Quevedo R, Shoffer M, Horng L and Oro AE: Receptor tyrosine phosphatase-dependent cytoskeletal remodeling by the hedgehog-responsive gene MIM/BEG4. J Cell Biol 168: 453-463, 2005.

17. Wang Y, Liu $\mathbf{J}$ and Smith E: Downregulation of missing in metastasis gene (MIM) is associated with the progression of bladder transitional carcinomas. Cancer Invest 25: 79-86, 2007.

18. Ma S, Guan XY, Lee TK and Chan KW: Clinicopathological significance of missing in metastasis B expression in hepatocellular carcinoma. Hum Pathol 38: 1201-1206, 2007. 